Supporting Information

\title{
An All-Carbon Negative Differential Resistance Nanodevice Using a Single Flake of Nanoporous Graphene
}

Reza Rahighi ${ }^{1}$, Omid Akhavan ${ }^{2}$, Ali Shayesteh Zeraati ${ }^{3}$, and Seyed Mehdi Sattari-Esfahlan ${ }^{4,5 *}$

${ }^{1}$ SKKU Advanced Institute of Nano-Technology (SAINT), Sungkyunkwan University, 2066, Seobu-ro, Jangan-gu, Suwon, Gyeonggi-do 16419, Republic of Korea

${ }^{2}$ Department of Physics, Sharif University of Technology, P.O. Box 11155-9161, Tehran, Iran ${ }^{3}$ Department of Chemical and Petroleum Engineering, University of Calgary, 2500 University Dr NW, Calgary, Canada T2N 1N4

${ }^{4}$ Research Institute of Advanced Materials (RIAM), Seoul National University, Seoul 08826, Republic of Korea

${ }^{5}$ Department of Materials Science and Engineering, Ajou University, Suwon 16499, Republic of Korea

* Corresponding author.

E-mail: smsisfahlanli@snu.ac.kr or smsattrie@gmail.com (S, M. Sattari-Esfahlan) 


\section{Estimation for the Number of Layers in PGFs and Comment on their Porous Structure}

The $\mathrm{L}_{h k l}$ (the mean dimensions of the crystallite perpendicular to the $(h k l)$ plane) can be determined by using Scherrer equation ${ }^{1}, \mathrm{~L}_{(h k l)}=\mathrm{k} \lambda / \beta \cos (\theta)$ where $\beta$ is the integral full widths at half maximum (in radians), $\lambda$ is wavelength of $\mathrm{X}$-ray, and $k$ is a constant $(0.89)$. The $\mathrm{d}_{(h k l)}$ (interspacing of layers) can also be calculated according to Bragg's relation ${ }^{2}, \mathrm{n} \lambda=2 \mathrm{~d}_{(h k l)} \sin (\theta)$ where $\mathrm{n}$ is an integer representing the order of diffraction peak. The number of layers in the highly

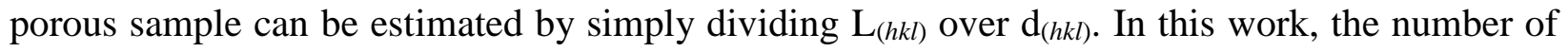
layers was found $\sim 6$, implying the presence of multi-layer sheets in the PGF structure, consistent with Raman spectrum analysis.

Synthesis of PGFs was performed under atmospheric pressure. Hence, the pressure of EWMs trapped in the pores was $\sim 1$ atm that is $10^{\wedge} 5 \mathrm{~Pa}$. After phase transition from liquid to gas state, the density usually increased up to 3 orders of magnitude. However, in the pores of our samples, the pores volume is nearly constant and so pressure would increase accordingly. This implies increase in the pressure of vaporized EWMs up to $\sim 10^{8} \mathrm{~Pa}$ in the pores, consistent with the conditions of supercritical water. It is worth reminding that such pressure is not so high in the realm of nanostructures. E.g. in nanobubbles, the subject of some other interesting topics, both theoretically and experimentally, ${ }^{3,4}$ the pressure can increase to the values as high as $\sim 1 \mathrm{GPa}$.

While performing the electrical measurement, the increase in voltage that causes higher current passing from the nanodevice, induces some heating as well. (The slight delay observed in the sharp NDR peak of our experiment is attributed to this.) The oxygen content of the experimental ambient condition is not high. Another oxygen source is therefore accountable for the NDR mechanism. In these conditions, the only supplier for oxygen is the EWMs trapped in the 
pores. Water in its normal state cannot supply high oxygen gas for reaction, while supercritical water with high effusion can do it.
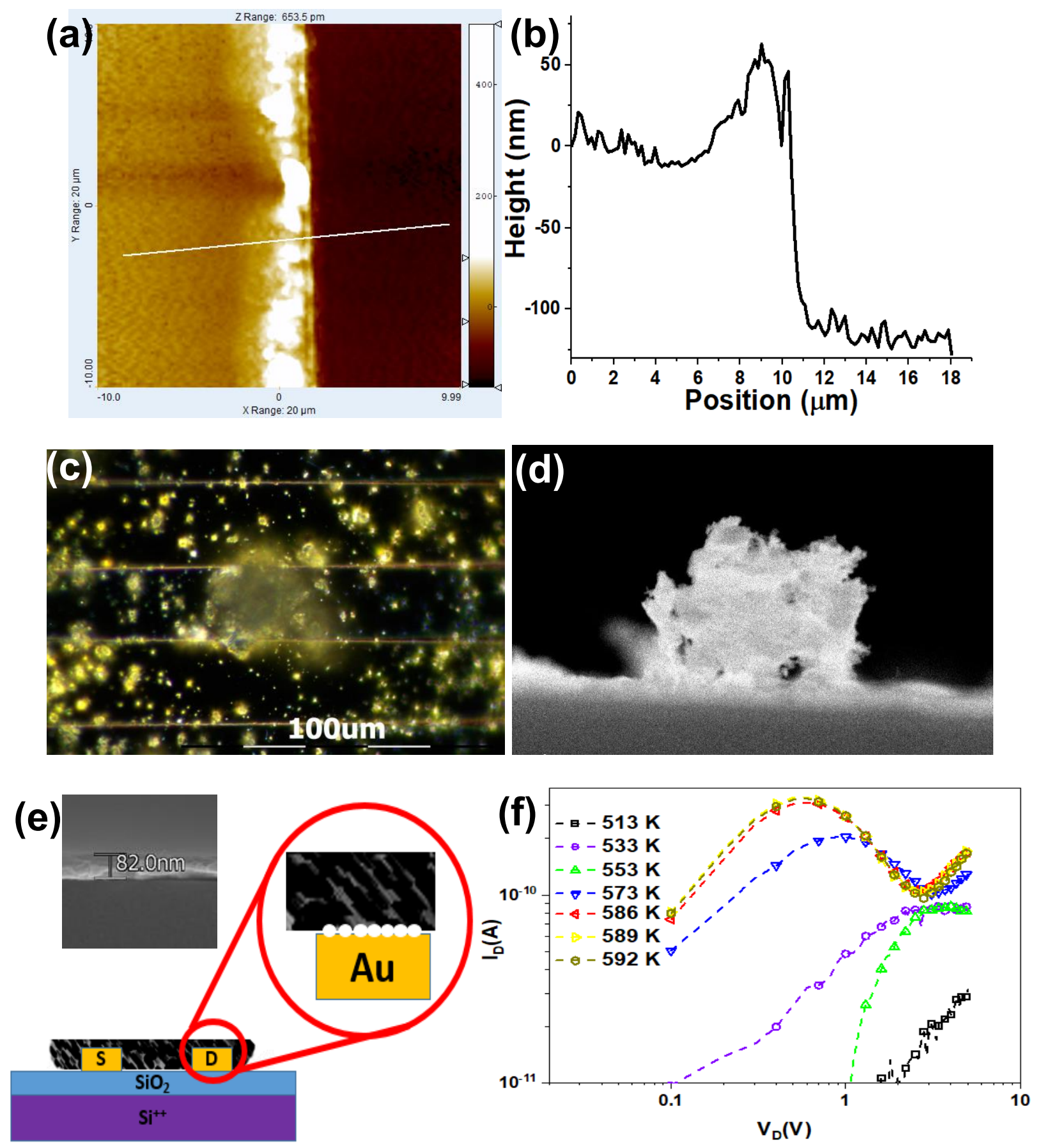
Figure S1. a) AFM image, b) height profile of the line, and c) Dark-field OM image of the as-prepared bottom-contacted nanodevice, d) cross-sectional SEM of the junction between a PGF and Au electrode after experiments, e) schematic illustration of probable phenomenon taking place at the junction between PGF and the gold electrodes (the inset shows $\sim 80 \mathrm{~nm}$ electrode thickness of deposited gold), and f) Log$\log$ presentation of data given in Fig 3c.

\section{Top -Contacted Configuration vs. Bottom-Contacted Nanodevice}

For top-contacted configuration, the Au electrodes (patterned by EBL) were deposited by using an e-beam evaporator on one isolated PGF flake, as seen in Figure S2a. General characteristics of two different sets of devices (investigated in this study) are compared in Table S2. In the top-contacted configuration, after mounting the PGF flake on the substrate, the Au electrodes were deposited, while the bottom contacted device was made by putting a single flake of the PGF between the two contacts. For the top-contacted device, the current was found linear and Ohmic, while for the bottom-contacted device, the current was tunneling along with the NDR effect. The higher current value in the top-contacted device was attributed to effective integration of gold atoms within the highly porous structure of PGF. Such more effective electrical contacts in 2D semiconductor-metal junctions is also reported by others..$^{5}$ This, of course, results in lower diffusion of ambient $\mathrm{O}_{2}$ gas into the reactive sites located near the GNPs. On the other hand, for the bottom-contacted device, there was no such effective metal deposition, resulting in looser

electrical contacts. Nevertheless, the less effective metal contacts in the bottom-contacted configuration provided higher possibility of oxygen diffusion into the pores, resulting in induction of the degenerate p-type region around the GNPs. 

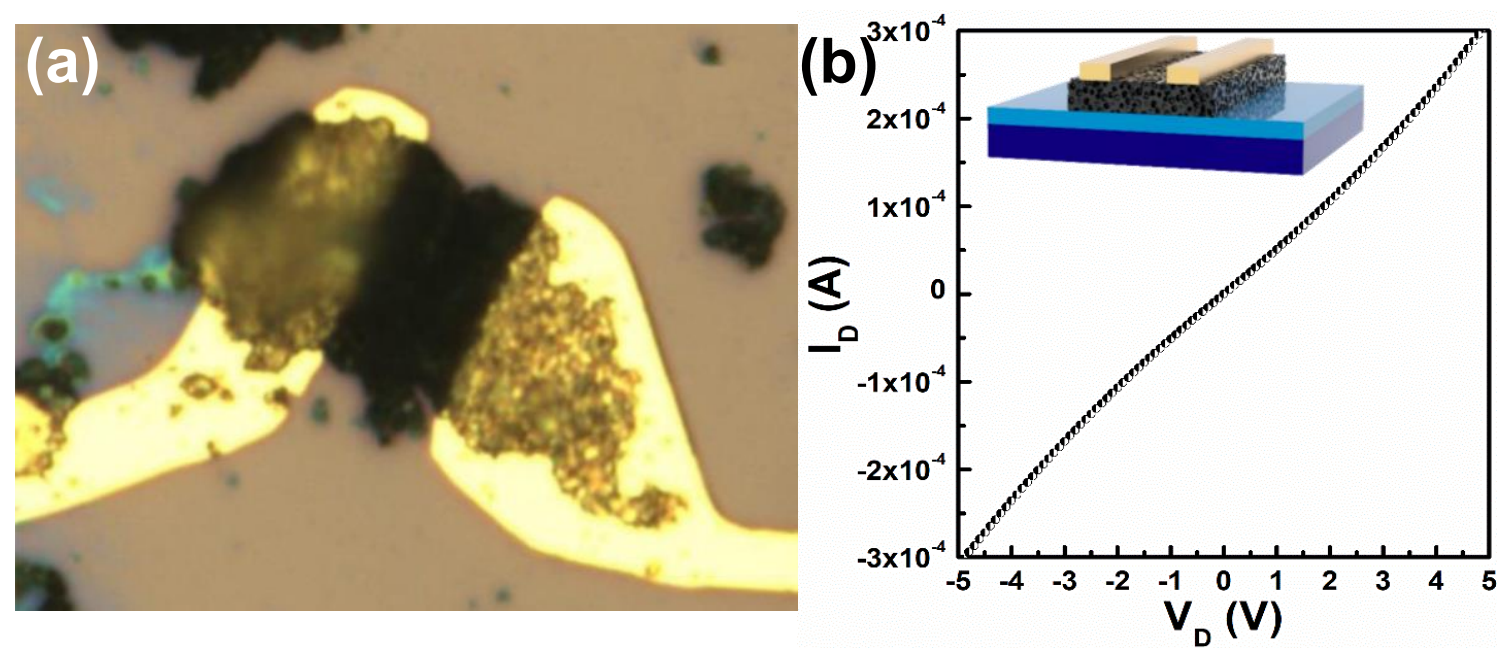

Figure S2. a) Bright-field OM image of the top-connected configuration, b) schematic of the fabricated nanodevice and its I-V characteristic measured at room temperature.

Table S1. Comparison between bottom-contact vs. top-contact devices

\begin{tabular}{ccc}
\hline Electrode & Top-contact & Bottom-contact \\
\hline Current & Linear & Non-linear \\
\hline Junction & Ohmic & Tunneling \\
\hline Current level & $\mu$ A range (high) & nA range (low) \\
\hline
\end{tabular}


(i)

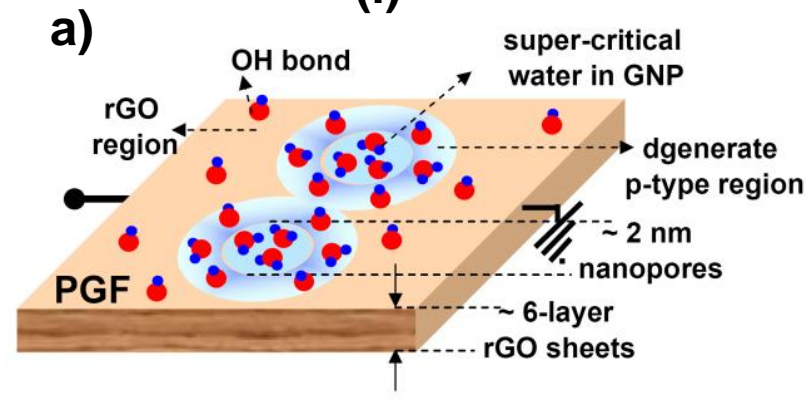

b) (ii)

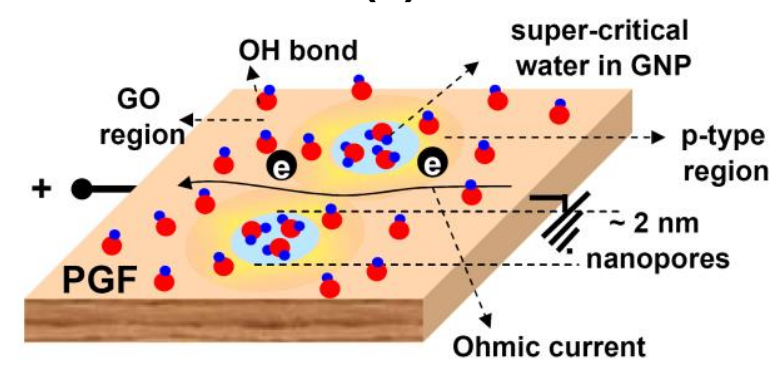

(ii)

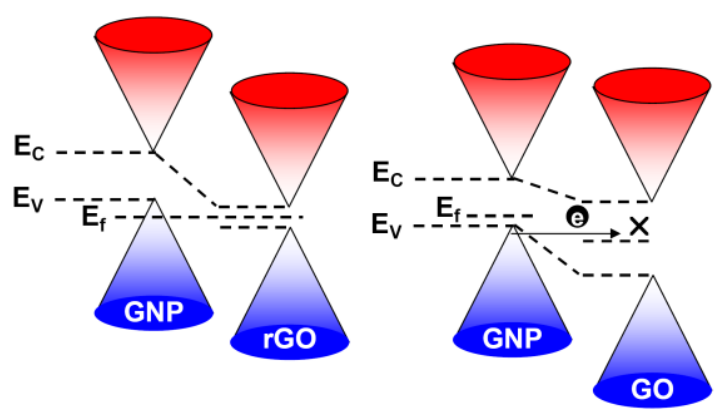

Figure S3. a) Schematic illustrations of a portion of the PGF with two neighboring aqueous pores at a high temperature inducing i) a degenerate p-type GNP/rGO heterojunction under applying no bias voltage and ii) a p-type GNP/GO heterojunction under applying the reverse bias voltage, b) the electron band structure of the temperature-induced i) degenerate p-type GNP/rGO heterojunction under applying no bias voltage and ii) p-type GNP/GO heterojunction under applying the reverse bias voltage.

\section{References}

(1) Pourmand, S.; Abdouss, M.; Rashidi, A. Fabrication of Nanoporous Graphene by Chemical Vapor Deposition (CVD) and Its Application in Oil Spill Removal as a Recyclable Nanosorbent. Journal of Industrial and Engineering Chemistry 2015, 22, 8-18. 
(2) Keshtkar, S.; Rashidi, A.; Kooti, M.; Askarieh, M.; Pourhashem, S.; Ghasemy, E.; Izadi, N. A Novel Highly Sensitive and Selective H2S Gas Sensor at Low Temperatures Based on SnO2 Quantum Dots-C60 Nanohybrid: Experimental and Theory Study. Talanta 2018, 188, 531-539. (3) Jannesari, M.; Akhavan, O.; Madaah Hosseini, HR.; Bakhshi, B. Graphene/CuO2 Nanoshuttles with Controllable Release of Oxygen Nanobubbles Promoting Interruption of Bacterial Respiration. ACS Applied Materials \& Interfaces 2020, 12, 35813-25.

(4) Jannesari M, Akhavan O, Hosseini HR. Graphene oxide in generation of nanobubbles using controllable microvortices of jet flows. Carbon 2018, 138, 8-17.

(5) Yang, Z.; Kim, C.; Lee, K. Y.; Lee, M.; Appalakondaiah, S.; Ra, C. H.; Watanabe, K.; Taniguchi, T.; Cho, K.; Hwang, E. A Fermi-Level-Pinning-Free 1d Electrical Contact at the Intrinsic 2D MoS2-Metal Junction. Advanced Materials 2019, 31, 1808231. 